\title{
Reasoning about orchestrations of web services using partial correctness
}

\author{
Alan Stewart ${ }^{1}$, Joaquim Gabarro ${ }^{2}$ and Anthony Keenan ${ }^{1}$ \\ ${ }^{1}$ School of EEE \& Computer Science, The Queen's University of Belfast, Belfast BT7 1NN, Northern Ireland, UK. \\ E-mail: a.stewart@qub.ac.uk, a.keenan@qub.ac.uk \\ 2 Dep. LSI, Universitat Politècnica de Catalunya, Jordi Girona, 1-3, 08034 Barcelona, Spain. \\ E-mail: gabarro@1si.upc.edu
}

\begin{abstract}
A service is a remote computational facility which is made available for general use by means of a wide-area network. Several types of service arise in practice: stateless services, shared state services and services with states which are customised for individual users. A service-based orchestration is a multi-threaded computation which invokes remote services in order to deliver results back to a user (publication). In this paper a means of specifying services and reasoning about the correctness of orchestrations over stateless services is presented. As web services are potentially unreliable the termination of even finite orchestrations cannot be guaranteed. For this reason a partial-correctness powerdomain approach is proposed to capture the semantics of recursive orchestrations.
\end{abstract}

Keywords: World Wide Web, Service, Specification, Orchestration, Orc, Partial correctness, Pre-orders, Fixedpoints, Powerdomains

\section{Introduction}

In a web environment developers can create applications by orchestrating a set of remote, distributed services. Internet-based software can be used to discover, organise and invoke remote services in order to carry out prespecified computations. For example, services are used in the cloud computational model to acquire both software and hardware resources. In [SGC06] it is argued that Orc [Mis04, MC07] provides appropriate combinators for constructing service-based computations. For example, Orc provides operators that can be used to time-out non-responsive services and reschedule computations elsewhere. In this paper a predicate-based [BvW, Heh84a, Hoa85] definition of Orc is given.

Conventionally, a specification $S$ is refined into a composition $P ; Q$ (or $P \| Q$ ) in such a way that correctness is preserved. An abstract specification may be transformed into code through a process of repeated refinement. In contrast orchestrations are constructed by assembling predefined services. One motivation for giving a predicate semantics to Orc is to enable the correctness of bottom-up designed orchestrations to be addressed.

Services are used as a basis for constructing a semantics for Orc in much the same way that assignment is used to provide a basis for an axiomatic semantics of an imperative programming language [Hoa69]. For example, a semantics for a conventional programming language can be derived by first defining assignment and then defining the language's compositional and recursive operators. In a conventional setting an assignment $x:=e$ is guaranteed to terminate provided that the expression $e$ is well-defined. Thus, (under the well-defined expression

Correspondence and offprint requests to: A. Stewart, E-mail: a.stewart@qub.ac.uk 
limitation above) a guarantee of program termination follows from proving that all recursive/iterative operators are finite (total correctness).

There is a fundamental distinction between a semantics of a standard programming language and a semantics of Orc; a conventional assignment (within the limitations mentioned above) terminates whereas a service call is, by its very nature, unreliable (and potentially divergent). A service is a software item (whose total correctness may have been established) which is made available in a web environment. The response behaviour of a service may vary, depending on the level of demand. If a large number of requests are made to a service $S$, within a given time interval, then the underlying hosting infrastructure is placed under stress. If demand exceeds the capacity of the hosting infrastructure then the response time of $S$ may be degraded, perhaps to the point of failure. In practice, orchestrations may call a number of (redundant) services having identical functionalities in order to provide a degree of robustness in the event of service failure. Even a simple non-recursive service call is potentially nonterminating; consequently, a partial-correctness setting is used to define the semantics of Orc expressions. For example, the semantics of a two-thread system which calls a BBC service and a CNN service in parallel must cater for the possibilities that both services are working, that exactly one service is operational or that both of the services are broken. The use of the lower powerdomain to define an orchestration captures this set of possible service behaviours.

Predefined Orc services can be specified as predicates. The approaches of [BvW, Heh84a, Heh84b, Hoa85, HH98] can be modified to provide a semantics for services and orchestrations. Three service variations are considered: stateless, shared state and private state. However, the reasoning framework presented here is designed primarily for orchestrations over stateless services. Such orchestrations arise in practice - e.g. certain kinds of computationally intensive scientific applications may be implemented as orchestrations which utilise (stateless) linear algebra services. Orc provides three combinators which can be used as glue for assembling pre-specified services into composite programs: | denotes parallel composition, $\gg$ denotes sequential composition and « denotes pruning composition. An orchestration publishes a bag of result values, $\theta$ : an orchestration with publication bag $\theta=\{a, b\}$ can informally be identified with a two-threaded computation, one thread publishing $a$ and the other $b$. The aim of this paper is to expose the nature of orchestration operators and to provide a formal semantics for reasoning about service-based computations.

Several other approaches have been used to give a semantics to Orc [DLSZ06, LZH10, HMM04, NS10, KCM06, VW08, WKCM08]: in both [LZH10] and [NS10] trace-based approaches are used to give a semantics to orchestrations. The trace approach of [LZH10] utilises a total-correctness UTP framework and has similarities with the approach taken in this paper. In [HMM04] orchestrations are mapped onto evaluation trees and the combinators of Orc are defined as tree operations. The work reported here differs in that it provides a mechanism for reasoning about orchestrations over concrete (pre-specified) services. Additionally, various operational and denotational semantics for Orc have been developed [KCM06, VW08, WKCM08]. An operational semantics provides details of service interactions that occur during an orchestration evaluation. In contrast, a predicatebased approach is concerned mainly with the values published by an orchestration ("what" rather than "how"). Although operational, denotational and predicate approaches have different motivations, the techniques have many similarities: for example, the mechanism for instantiating variables that is used in this paper is essentially that used in [WKCM08]. The predicate-based semantics given here offers a direct style of reasoning about orchestration input/output behaviour. In addition, a semantics for recursive orchestrations is developed.

A predicate-based framework for specifying services is presented in Sect. 2. Informal and formal (stateless) semantics of Orc are given in Sects. 3 and 4, respectively. Powerdomains are used to derive a semantics of recursive Orc expressions in Sect. 5. In Sect. 6 some suggestions for future work are outlined.

\section{Services}

A service is a remote, potentially unreliable, computational facility. Services can be stateless or stateful - for example, the service call cloud_execute $(C, d)$ returns the result of remotely executing code $C$ on data $d$ while the service database $D B L P$ utilises a dynamic (state-based) database. A service is called asynchronously from a computational thread. At some later time the service may publish a response [WKCM08]. Service calls may be unsuccessful for a number of reasons: data supplied in a service call may be inappropriate, a service-based computation may diverge, a service may not have the capacity to process simultaneously the current volume of calls, or communications may be "lost" on faulty networks. 


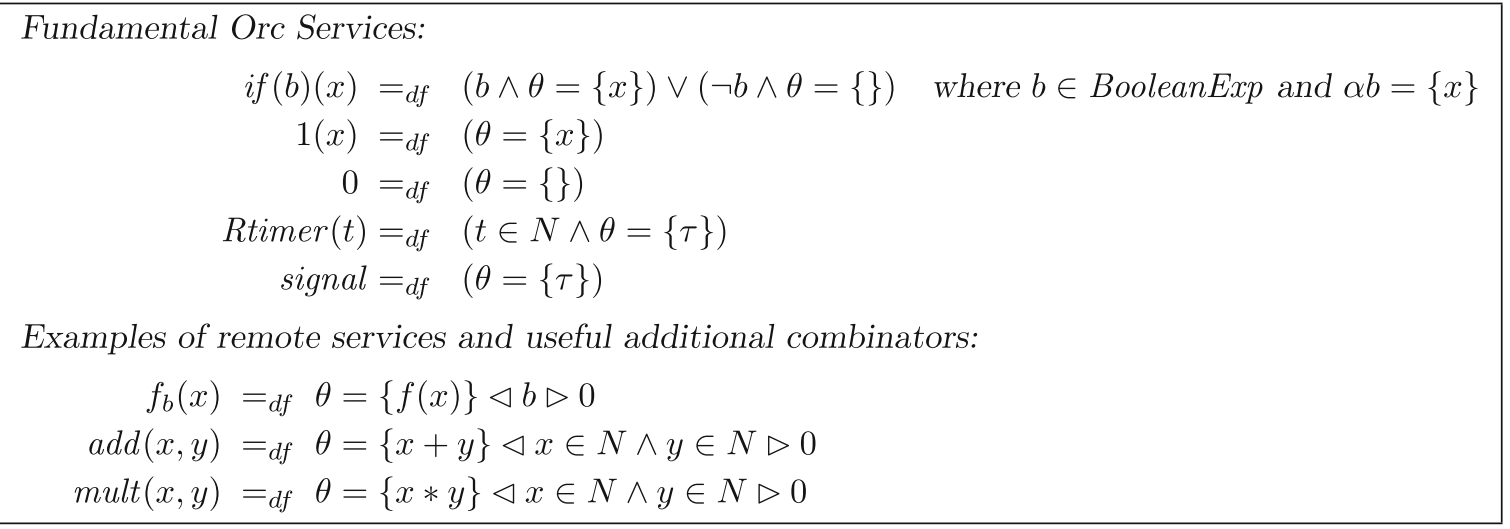

Fig. 1. Examples of Orc services and orchestrations

Services are specified using predicates (in much the same way that programs are in both UTP [HH98] and predicative programming [Heh84a, Heh84b]). In the remainder of this paper a bag or multiset is denoted using conventional set notation - $\{a, a\}$ denotes a bag with two occurrences of element $a$. The alphabet of a predicate $P, \alpha P$, comprises the set of variables that occur free in $P$. The alphabet $\alpha P$ is partitioned into input variables, in $\alpha P$, and the distinguished output variable $\theta$.

Definition 1 (stateless service) The stateless service specification $S(x)={ }_{d f} P$ identifies the name $S$ with the relation $\{(x, \theta) \mid P\}$ where $x$ and $\theta$ are free variables of $P$ and where $\theta$ denotes either a singleton output bag (of results) published by $S$ or an empty bag (in the case of non-termination). Service $S$ is instantiated by giving a binding for the input variable $x$ :

$$
S(a)={ }_{d f} P_{a}^{x}
$$

where $P_{a}^{x}$ is $P$ with all free occurrences of $x$ replaced by $a$. The service call $S(a)$ can publish any result bag $r$ where $r \in\left\{\theta \mid P_{a}^{x}\right\}$.

Definition 2 (shared state service) Services can have shared states which can be updated by a wide range of usersfor example, the state of an auction service can be changed by any registered user. The stateful service specification $S_{s}(x)=d_{d f} Q$ identifies the name $S_{s}$ with the relation $\left\{\left(x, \sigma, \sigma^{\prime}, \theta\right) \mid Q\right\}$ where $x$ is an input variable, $\sigma$ and $\sigma^{\prime}$ are, respectively, before and after state variables and $\theta$ is the output variable. From the point of view of an individual user a shared state may have no persistence. A call to $S_{s}$ is identified with the predicate $\exists \sigma, \sigma^{\prime}$. $Q$ since the current state of a service may be in dynamic flux. Service $S_{s}$ is instantiated by giving a binding for the input variable $x$ :

$$
S_{s}(a){ }_{d f} \exists \sigma, \sigma^{\prime} \cdot Q_{a}^{x}
$$

This instantiation rule makes no assumptions about the initial state of $S_{s}$ (other than state properties that are guaranteed by $Q$ ). The service call $S_{s}(a)$ can publish any result bag $r$ where $r \in\left\{\theta \mid \exists s^{\prime}, s . Q_{a}^{x}\right\}$.

The use of existential quantifiers captures the myriad of possibilities that may arise when a group of users interact concurrently with state-based services. In practice, state invariants may be added to stateful services to limit the degree of non-determinism.

Definition 3 (private state service for a user $u$ ) The Amazon book service provides a user $u$ with an individual shopping basket - the private state of this basket is only visible to (and modifiable by) $u$. A service with private state $\sigma$ is specified as: $S_{p}(x)={ }_{d f} R\left(x, \sigma, \sigma^{\prime}, \theta\right)$ where $x$ is an input variable, $\sigma$ and $\sigma^{\prime}$ are, respectively, before and after private state variables and $\theta$ is the output variable. A private state for $u$ persists until it is altered by $u$. An instantiation of a private state service

$$
S_{p}(a)={ }_{d f} \quad R_{a}^{x}
$$

defines both a state transformation and a publication bag. The focus of this paper is on orchestrations over stateless services. However, some consequences of employing orchestrations over services with private states are considered in Sect. 6. 
Orc incorporates a small number of fundamental services - see Fig. 1 . The vacuous service 0 can never publish - it models a service call with invalid parameters or a call to a divergent service. 0 is denoted by predicate $\theta=\{\}$. Service 1 always returns its argument. Service $i f(b)(x)$ returns argument $x$ if it satisfies Boolean expression $b$; otherwise if $(b)$ is silent. ${ }^{1}$ The definition of if can be extended to multiple inputs - such as $(x, y)$-in an obvious way. The service Rtimer $(t)$ publishes a signal $\tau$ after $t$ time units have elapsed. An orchestration may additionally call upon a wide range of remote (unreliable) services (see Fig. 1 for some typical examples). Service $f_{b}$ applies function $f$ to its input data if $b$ holds - otherwise $f_{b}$ is silent (i.e. behaves as 0 ). add and mult are concrete instances of $f_{b}$.

In practice it is often convenient to use a generalised form of selection; the orchestration $\left(P_{1} \triangleleft b \triangleright P_{2}\right)$ calls service $P_{1}$ if $b$ holds and calls $P_{2}$ otherwise.

$$
P \triangleleft b \triangleright Q=_{d f}(b \wedge P) \vee(\neg b \wedge Q)
$$

Non-determinism is used later to provide the basis for a semantic framework for Orc - the notation $P \sqcup Q$ is used to denote a non-deterministic choice between orchestrations $P$ and $Q$.

$$
P \sqcup Q={ }_{d f} P \vee Q
$$

CHAOS (or true) is the most non-deterministic orchestration which can publish any bag of values or diverge.

Orchestration predicates are required to specify output publication bags. Thus, 0 is specified by the predicate $\theta=\{\}$ rather than false. To simplify matters false is "removed" from the class of admissible predicates by introducing a healthiness condition-predicate $P$ is healthy if

$\mathbf{H} \quad[\exists \theta . P]$

Here the brackets [...] denote universal quantification over all free variables. For example, the predicate $x \neq 0 \wedge \theta=\{y \div x\}$ is not healthy since no publication bag is defined when $x=0$.

Example 1 Service 1(a) publishes its argument $a$ when called. The predicate $\theta=\{y \div x\} \triangleleft x \neq 0 \triangleright \theta=\{\}$ specifies a division service $\operatorname{DIV}(y, x)$. A service specification with two (or more) inputs can be partially instantiated: for example, $\operatorname{add}(3, y)$ is denoted by $(y \in N \wedge \theta=\{3+y\}) \vee(\neg(y \in N) \wedge \theta=\{\})$.

In practice, services are often made available on networks which have the potential to become stressed, at certain points, due to over-demand. In such circumstances service calls have the potential to fail. The behaviour of an addition service which is made available on an unreliable environment can be specified as:

$$
a d d_{u}(x, y)=_{d f} \operatorname{add}(x, y) \vee \theta=\{\}
$$

\section{Orc}

The operators of Orc act as a glue for cementing service calls together. Parallel composition (|) is used to evaluate multiple threads simultaneously, sequential composition $(\gg)$ can be used to sequence single or multiple threads and pruning composition $(\ll)$ can be used to non-deterministically select one publication from a set of threads. An otherwise operator $(>)$ has been proposed recently [Kit09, LZH10] which activates a second orchestration in the event that a first orchestration terminates without publishing. The formal syntax of Orc expressions [Mis04] is given in Fig. 2. An informal description of each of the operators is given below.

Evaluation of the composition $P \mid Q$ executes $P$ and $Q$ in parallel and publishes some interleaving of the publications of $P$ and $Q$. Thus, the publication bag of $P \mid Q$ is the union of the publication bags of $P$ and $Q$. The alphabet of $P \mid Q$ is in $\alpha P \cup i n \alpha Q$. Evaluation of the expression $\operatorname{add}(a, a) \mid$ add $(c, c)$ publishes the values $2 a$ and $2 c$ - in the formal model in $\S 4$ expression $a d d(a, a) \mid \operatorname{add}(c, c)$ is associated with the bag $\{2 a, 2 c\}$.

Sequential composition, $\gg$, is used to sequence service calls or expressions The alphabet of $P>x>Q$ is in $\alpha P \cup($ in $\alpha Q-\{x\})$. The sequencing of service calls is analogous to conventional sequential composition: for example, evaluation of $1(a)>x>$ add $(x, b)$ publishes the output $a+b$. Here the publication value produced by $1(a)$ is bound to $x$ and subsequently used to instantiate $a d d(x, b)$. If $x$ is free in $P$ then it is also free in $P>x>Q$; however use of the linking binding $(>x>$ ) within $P>x>Q$ means that all occurrences of $x$ within $Q$ are not free. ${ }^{2}$ More generally, an orchestration $E$ may publish a bag of results; an evaluation of $E>x>F$ spawns new parallel threads, $F_{v}^{x}$, for each value $v$ published by $E$. For example, an evaluation of $(1(a) \mid 1(c))>x>a d d(c, x)$ spawns two parallel threads, $a d d(c, a)$ and $a d d(c, c)$ and, subsequently publishes both $c+a$ and $2 c$.

\footnotetext{
1 Note: if is defined in [Mis04, MC07] to return a signal.

2 An expression such as $R>x>(P>x>Q)$ can be rewritten as $R>z>\left(P_{z}^{x}>x>Q\right)$ where $z$ is a fresh variable name.
} 


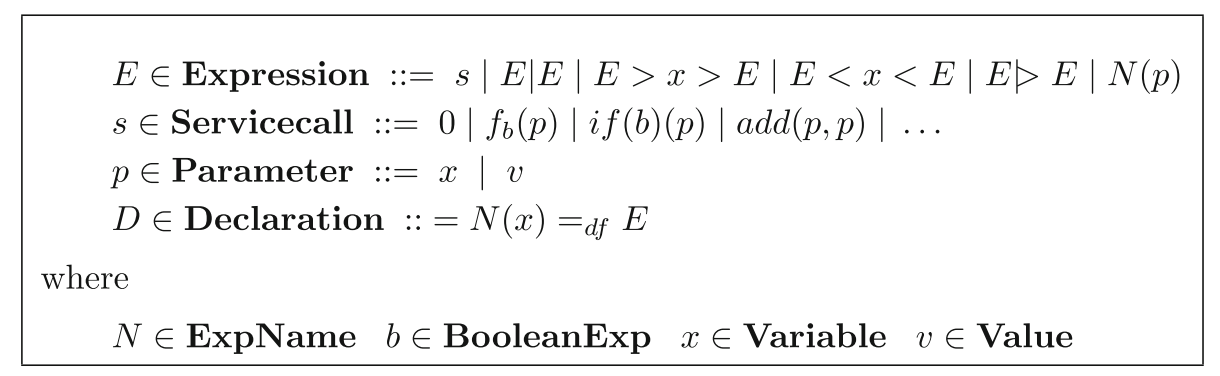

Fig. 2. Syntax of Orc

Pruning composition provides a means of defining time-outs and merges: the composition $Q<x<P$ nondeterministically selects one output generated from the evaluation of $P$ to generate a binding for $x$ while at the same time evaluating $Q$. When a binding for $x$ becomes available all uncompleted threads of $P$ are terminated. Evaluation of $Q$ may become partially blocked until a binding for $x$ is generated at which point evaluation of uncompleted threads within $Q$ is resumed. For example, the orchestration $1(x)<x<\left(f_{b}(a) \mid\right.$ Rtimer $\left.(t)\right)$ publishes $f(a)$, if service $f_{b}$ responds before $t$ time units elapse; otherwise the signal $\tau$ is published. The orchestration $\left(g_{b}(a) \triangleleft x=\tau \triangleright 1(x)\right)<x<\left(f_{b}(a) \mid\right.$ Rtimer $\left.(t)\right)$ calls service $g$ if a previous call to $f$ fails to respond quickly enough. The nested pruning composition

$$
(1(y, z)<y<\operatorname{mult}(4,4))<z<\operatorname{mult}(5,5)
$$

computes the tuple $(16,25)$. In the orchestration $Q<x<P$ all threads within $P$ may diverge; in this case those threads within $Q$ that are independent of $x$ are evaluated while the remaining threads of $Q$ are permanently blocked. The expression $Q \downarrow x$ denotes that part of $Q$ (the $x$-residual) that is independent of $x . Q \downarrow x$ is defined over the structure of Orc expressions:

$$
\begin{aligned}
s(z) \downarrow x & =d f(0 \triangleleft x \in \alpha s(z) \triangleright s(z)) \\
(E \mid F) \downarrow x & ==_{d f}(E \downarrow x) \mid(F \downarrow x) \\
(E>x>F) \downarrow x & ==_{d f}(E \downarrow x)>x>F \\
(E>z>F) \downarrow x & ==_{d f}(E \downarrow x)>z>(F \downarrow x) \\
(E<x<F) \downarrow x & ==_{d f} E<x<(F \downarrow x) \\
(E<z<F) \downarrow x & ={ }_{d f}(E \downarrow x)<z<(F \downarrow x)
\end{aligned}
$$

The definition of $x$-residual assumes that services use a call-by-value evaluation mechanism.

The otherwise combinator $P \gg Q$ behaves as $P$ if either $P$ publishes or is silent due to non-termination; if $P$ terminates without publishing then $Q$ is activated [Kit09]. For example, consider the orchestration

$$
C N N(d) \gg B B C(d)
$$

which utilises the digital newspaper services $C N N$ and $B B C$. Both services return a digital newspaper for a given date, $d$, if possible. If no digital newspaper is currently available for date $d$ then the $C N N$ and $B B C$ services terminate without publication. The expression $C N N(d) \triangleright B B C(d)$ "raises an exception" if $C N N$ fails to publish; in these circumstances the $B B C$ service is activated.

A semantics for $>$ can be provided in a setting in which termination observations are possible - see, for example, the trace semantics in [LZH10]. The behaviour of $P \mid>Q$ in a unreliable service environment gives rise to nondeterministic behaviour. ${ }^{3}$

\footnotetext{
3 It is also possible to raise exceptions in Orc by means of "no publication" signals. For example, suppose that $\overline{C N N(d)}$ is a digital newspaper service which publishes a $\tau$ signal if no newspaper is available for date $d$. The expression $\overline{C N N(d)}>x>(B B C(d) \triangleleft x=\tau \triangleright 1(x))$ raises an exception if $C N N$ cannot publish a digital newspaper. Non-judicious use of such $\tau$ signals has the potential to cause "non-publishing" threads to be retained at the expense of publishing threads inside pruning compositions.
} 


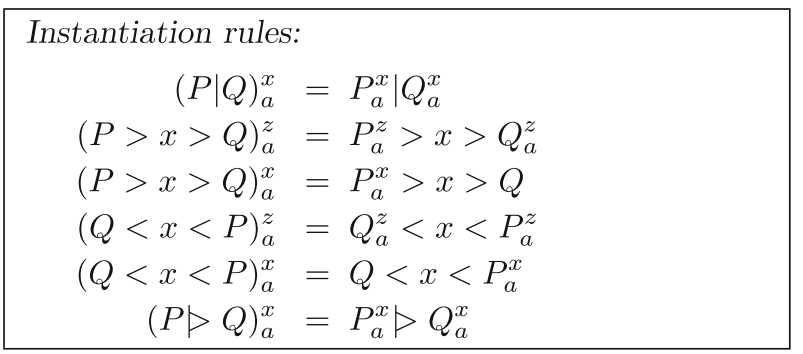

Fig. 3. Rules for instantiating Orc expressions

A declaration binds a name to an Orc expression. The declaration

timed_add $(x, y, t)=_{d f} 1(z)<z<(\operatorname{add}(x, y) \mid \operatorname{Rtimer}(t))$

associates the name timed_add with an expression which implements a time-out mechanism. Declarations can be instantiated in a similar way to services - the formal definition of expression instantiation is given in Fig. 3 .

Declarations may be recursive. For example:

$a d d^{*}(x, y)=_{d f}$ timed_add $(x, y, 10)>z>\left(a d d^{*}(x, y) \triangleleft z=\tau \triangleright 1(z)\right)$

repeatedly calls $a d d$, with a time-out mechanism, until it responds.

\section{Orchestration semantics for stateless services}

In this section a means of reasoning about orchestrations over stateless services is described. To this end predicatebased definitions of the Orc combinators are given - this allows an Orc expression over pre-defined services to be equated with a relation from inputs to publication bags. For example, an orchestration which is identified with the predicate $\theta=\{a\} \vee \theta=\{a, c\}$ can publish either $a$ or an interleaving of $a$ and $c$.

The parallel composition of $P$ and $Q$ forms the bag union of the publication bags of $P$ and $Q$. In the definition of $P \mid Q$ the publications of (i) $P$ (ii) $Q$ and (iii) $P \mid Q$ are distinguished as follows: $P \mid Q$ publishes $\theta$ while the publication bags of $P$ and $Q$ are renamed $r_{1}$ and $r_{2}$, respectively. This renaming of component publication bags is typical of the remaining semantic definitions.

The sequential composition $P>x>Q$ spawns instantiations $Q_{v}^{x}$ for each value $v$ in the publication bag of $P$-in effect $P>x>Q$ constructs a set of sequenced threads. In the definition of $P>x>Q$ instantiation is generalised from values to bags of values: if $b$ is a publication bag then $Q_{b}^{x}$ denotes a parallel composition of conventional (single-valued) instantiations $\left.\right|_{y \in b} Q_{y}^{x}$.

Evaluation of $Q<x<P$ proceeds by executing $P$ and $Q$ in parallel (the evaluation of some threads within $Q$ may need to be delayed until a binding for $x$ is generated by $P$ ): the first output published by $P$ is used to instantiate $x$; at this point evaluation of $P$ is terminated and evaluation of $Q$ is completed. If $P$ diverges then evaluation of $Q<x<P$ evaluates those threads of $Q$ that are independent of $x$, viz $Q \downarrow_{x}$; otherwise $Q<x<P$ behaves as $Q$ instantiated by some element taken from the publication bag of $P$. One essential difference between the operators $\gg$ and $\ll$ is that sequential composition uses bag instantiation whereas pruning composition non-deterministically chooses an element from a bag before using conventional single-value instantiation.

Evaluation of $P \mid>Q$ proceeds by first evaluating $P$. If $P$ publishes then $P \mid>Q$ behaves as $P$. If $P$ is silent by design (i.e. $P$ terminates without publishing) then an exception is raised and $Q$ is invoked. However, if $P$ is silent and fails to terminate then $P \gg Q$ remains silent. As termination is unobservable in a partial semantics the silence of $P$ results in non-determinism (i.e. $P \mid>Q$ behaves as either 0 or $Q$ ). The combinators of Orc are formally defined in Fig. 4. The following examples illustrate combinator effects. Suppose that

$$
S q(x)=_{d f} \theta=\left\{x^{2}\right\} \triangleleft x \in N \triangleright \theta=\{\}, \quad \operatorname{Cube}(x)=_{d f} \theta=\left\{x^{3}\right\} \triangleleft x \in N \triangleright \theta=\{\}
$$

are services which compute the square and cube of an input, respectively. Then:

$$
\begin{aligned}
(S q(x) \mid \text { Cube }(x)) & =\theta=\left\{x^{2}, x^{3}\right\} \triangleleft x \in N \triangleright \theta=\{\} \\
(S q(x) \mid \text { Cube }(x))>y>1(x, y) & =\left(\theta=\left\{\left(x, x^{2}\right),\left(x, x^{3}\right)\right\} \triangleleft x \in N \triangleright \theta=\{\}\right. \\
(1(x) \mid 1(y))<y<(S q(x) \mid \text { Cube }(x)) & =\left(\theta=\left\{\left(x, x^{2}\right)\right\} \vee \theta=\left\{\left(x, x^{3}\right)\right\}\right) \triangleleft x \in N \triangleright \theta=\{x\}
\end{aligned}
$$




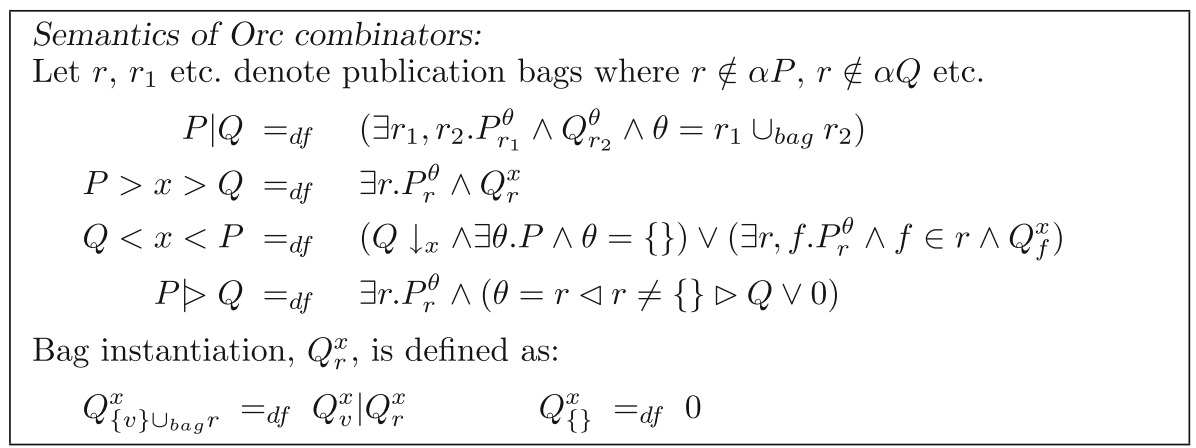

Fig. 4. Semantics of the Orc combinators $\mid, \gg, \ll$ and $\mid>$

Orchestrations are often implemented in unreliable multi-threading environments (see section 3.2 [MC07]). Any thread which makes a call to a remote service may fail. One approach to reasoning about orchestrations acting in unreliable environments is partial correctness. Suppose that $S q_{u}$ and $C u b e_{u}$ are versions of the square and cube services, respectively, which are made available on unreliable web environments:

$$
S q_{u}(x)=_{d f}\left(\theta=\left\{x^{2}\right\} \wedge x \in N\right) \vee \theta=\{\}, \quad \operatorname{Cube}_{u}(x)=_{d f}\left(\theta=\left\{x^{3}\right\} \wedge x \in N\right) \vee \theta=\{\}
$$

The behaviour of $\left(S q_{u}(x) \mid \mathrm{Cube}_{u}(x)\right)>y>1(x, y)$ is:

$$
\left(S q_{u}(x) \mid \mathrm{Cube}_{u}(x)\right)>y>1(x, y)=\left(\theta=\{\} \vee \theta=\left\{\left(x, x^{2}\right)\right\} \vee \theta=\left\{\left(x, x^{3}\right)\right\} \vee \theta=\left\{\left(x, x^{2}\right),\left(x, x^{3}\right)\right\}\right)
$$

Thus, if $\theta$ is a publication of an orchestration $R$ in an unreliable web-environment then so to is $\theta^{\prime}$, where $\theta^{\prime} \subseteq \theta$. Partial correctness is treated more fully in Sect. 5.

The definitions of the Orc combinators can be used to derive algebraic orchestration laws. Two distinct sets of laws can be constructed:

- stateless orchestration laws: two orchestrations are equivalent if their publications are identical;

- stateful orchestration laws: two orchestrations are equivalent if they have the same publication bags and the same state transformations.

The use of bag union in the definition of parallel composition has the consequence that $P \neq P \mid P$. The behaviour of combinators under service failure is captured by the following laws:

$$
\begin{aligned}
P \mid 0 & =P \\
0>x>Q & =0 \\
Q<x<0 & =Q \downarrow_{x}
\end{aligned}
$$

In addition, the following laws hold for stateless services:

$$
\begin{aligned}
& P>x>0=0 \\
& 0<x<Q=0
\end{aligned}
$$

The distribution law

$$
(P \mid Q)>x>R=(P>x>R) \mid(Q>x>R)
$$

follows from the definition of bag instantiation: $R_{b \cup c}^{x}=R_{b}^{x} \mid R_{c}^{x}$. For orchestrations over stateless services the reverse distribution property also holds:

$$
P>x>(Q \mid R)=(P>x>Q) \mid(P>x>R)
$$

However, if $P$ is stateful then this law is no longer valid [HMM04] since $P$ appears once on the left hand side and twice on the right hand side. The relationship between parallelism, pruning composition and non-determinism is expressed by the following conditional law:

$$
\frac{\forall \theta \cdot((P \Rightarrow \theta \neq\{\}) \wedge(Q \Rightarrow \theta \neq\{\})) \vee \exists \theta .(P \wedge Q \wedge \theta=\{\})}{R<x<(P \mid Q)=(R<x<P) \sqcup(R<x<Q)}
$$


Here $P$ and $Q$ have the same publication potential (i.e. both $P$ and $Q$ must be guaranteed to publish or both must have the potential to fail). Pruning composition does not distribute through parallel composition, even for stateless services:

$$
(Q \mid R)<x<P \neq(Q<x<P) \mid(R<x<P)
$$

For example, let $A B=(\theta=\{a, b\})$. Then $(Q \mid R)<x<A B=\left(Q_{a}^{x} \mid R_{a}^{x}\right) \vee\left(Q_{b}^{x} \mid R_{b}^{x}\right)$ whereas

$$
(Q<x<A B) \mid(R<x<A B)=\left(Q_{a}^{x} \mid R_{a}^{x}\right) \vee\left(Q_{b}^{x} \mid R_{b}^{x}\right) \vee\left(Q_{a}^{x} \mid R_{b}^{x}\right) \vee\left(Q_{b}^{x} \mid R_{a}^{x}\right)
$$

Orc has a full set of distribution laws for non-deterministic choice:

$$
\begin{aligned}
(P \sqcup Q) \mid R & =(P \mid R) \sqcup(Q \mid R) & & (\sqcup D 1) \\
P>x>(Q \sqcup R) & =(P>x>Q) \sqcup(P>x>R) & & (\sqcup D 2) \\
(P \sqcup Q)>x>R & =(P>x>R) \sqcup(Q>x>R) & & (\sqcup D 3) \\
R<x<(P \sqcup Q) & =(R<x<P) \sqcup(R<x<Q) & & (\sqcup D 4) \\
(Q \sqcup R)<x<P & =(Q<x<P) \sqcup(R<x<P) & & (\sqcup D 5)
\end{aligned}
$$

For example, law $\sqcup D 5$ can be proved as follows:

$$
\begin{aligned}
& (Q \sqcup R)<x<P= \\
& \left((Q \sqcup R) \downarrow_{x} \wedge \exists \theta . P \wedge \theta=\{\}\right) \vee\left(\exists r . \exists f . P_{r}^{\theta} \wedge f \in r \wedge(Q \sqcup R)_{f}^{x}\right)= \\
& \left(Q \downarrow_{x} \wedge \exists \theta . P \wedge \theta=\{\}\right) \vee\left(R \downarrow_{x} \wedge \exists \theta . P \wedge \theta=\{\}\right) \\
& \quad \vee\left(\exists r . \exists f . P_{r}^{\theta} \wedge f \in r \wedge Q_{f}^{x}\right) \vee\left(\exists r . \exists f . P_{r}^{\theta} \wedge f \in r \wedge R_{f}^{x}\right)= \\
& \left(Q \downarrow_{x} \wedge \exists \theta . P \wedge \theta=\{\}\right) \vee\left(\exists r . \exists f . P_{r}^{\theta} \wedge f \in r \wedge Q_{f}^{x}\right) \vee\left(R \downarrow_{x} \wedge \exists \theta . P \wedge \theta=\{\}\right) \\
& \quad \vee\left(\exists r . \exists f . P_{r}^{\theta} \wedge f \in r \wedge R_{f}^{x}\right)= \\
& (Q<x<P) \sqcup(R<x<P)
\end{aligned}
$$

\section{Recursive declarations}

A declaration is a binding of a name to an orchestration expression: the declaration $d(x)=_{d f} E$ binds the name $d$ and the formal parameter $x$ to the expression $E$. If $d \notin \alpha E$ then declaration $d$ is non-recursive and so $d(a)=E_{a}^{x}$. Otherwise, $d$ has the recursive form $d(x)=E(d)$. In this case a semantics is given to $d$ by treating $d(x)=E(d)$ as a fixed-point equation [HH98]. Here the lower powerdomain (see below) is used as a basis for generating a chain of improving approximations $\left[P_{i} \mid i \geq 0\right]$ to $d$. Each predicate $P_{i}$ is identified with a relation from inputs to publications. The meaning of $d$ is the least upper bound of the approximation sequence $\left[P_{i} \mid i \geq 0\right]$.

Recursive declarations can be used to define both threads (which publish single values) and systems of threads. The declaration

$$
f a c(x)=_{d f} \theta=\{1\} \triangleleft x=0 \triangleright(f a c(x-1)>y>\operatorname{mult}(x, y))
$$

corresponds to a factorial computation. The declaration

$$
f^{*}(x)=\left(f^{*}(x) \triangleleft z=\tau \triangleright 1(z)\right)<z<(f(x) \mid \operatorname{Rtimer}(t))
$$

repeatedly calls service $f$, using a time-out mechanism, until it responds.

The declaration $S(n)$ where

$$
\begin{aligned}
& S(0)={ }_{d f} 1(0) \\
& S(n)={ }_{d f} 1(n) \mid S(n-1) \text { if } n>0
\end{aligned}
$$

spawns $n$ threads and publishes the result bag $\theta=\{n, n-1, \ldots, 0\}$. The declaration

$$
T(n)=_{d f} 1(n) \mid T(n+1)
$$

spawns an unbounded number of threads - note that $T(n)$ could not be implemented in practice. 
A solution to the recursive declaration $d=E(d)$ can be found using an appropriate partial (or pre-) order $\sqsubseteq$ (over relations) on which the orchestration operators are monotonic. An operation $f$ is monotonic on $\sqsubseteq$ if for arbitrary $P$ and $Q$ :

$$
P \sqsubseteq Q \Rightarrow f(P) \sqsubseteq f(Q)
$$

Powerdomains offer a means of ordering multi-threaded systems. There are three powerdomains (see $\$ 5$ in [GS90]):

- the lower or Hoare powerdomain; here predicates $P$ and $Q$ are ordered by:

$$
P \sqsubseteq_{l} Q=_{d f}\left[\forall \theta . P \Rightarrow \exists \theta^{\prime} . Q_{\theta^{\prime}}^{\theta} \wedge \theta \subseteq_{b a g} \theta^{\prime}\right]
$$

$P$ is weaker than $Q$ if every result published by $P$ is covered from above (i.e. is also published by $Q$ ). The lower powerdomain ordering does not distinguish orchestrations by termination properties: $P \sqsubseteq_{l} P \sqcup 0$ and $P \sqcup 0 \sqsubseteq_{l} P$ (i.e. $\sqsubseteq_{l}$ can be used to construct a partial-correctness semantics).

- the upper or Smyth powerdomain:

$$
P \sqsubseteq_{u} Q=_{d f}\left[\forall \theta^{\prime} . Q_{\theta^{\prime}}^{\theta} \Rightarrow \exists \theta \cdot P \wedge \theta \subseteq_{b a g} \theta^{\prime}\right]
$$

$P$ is weaker than $Q$ if every result published by $Q$ is covered from below. The upper powerdomain does not distinguish orchestrations whose termination is not guaranteed: $0 \sqsubseteq_{u} P \sqcup 0$ and $P \sqcup 0 \sqsubseteq_{u} 0$ (i.e. total correctness). The strong termination requirements of $\sqsubseteq_{u}$ make it unsuitable for giving a semantics to orchestrations over unreliable services.

- the convex or Plotkin powerdomain:

$$
P \subseteq_{c} Q=_{d f}\left[\left(\forall \theta \cdot P \Rightarrow \exists \theta^{\prime} . Q_{\theta^{\prime}}^{\theta} \wedge \theta \subseteq_{b a g} \theta^{\prime}\right) \wedge\left(\forall \theta^{\prime} . Q_{\theta^{\prime}}^{\theta} \Rightarrow \exists \theta \cdot P \wedge \theta \subseteq_{b a g} \theta^{\prime}\right)\right]
$$

The ordering $\sqsubseteq_{c}$ is known as the Egli-Milner ordering and ensures that every publication is covered from both below and above. The Plotkin powerdomain has the finest granularity with $0 \sqsubset_{u}(P \sqcup 0) \sqsubset_{u} P$. This level of equality is unsuited to describing orchestrations in uncertain environments. Additionally, the pruning combinator $\ll$ is non-monotonic on $\sqsubseteq_{c}$. For example, if

$$
A=(\theta=\{a\}), B=(\theta=\{b\}) \text { and } A B=(\theta=\{a, b\})
$$

then $A \sqsubseteq_{c} A B$ but $(1(z)<z<A)=A$ is not weaker than $(1(z)<z<A B)=A \vee B$ since $B$ is not covered from below.

Relation $\subseteq_{l}$ is used to define the notion of partial correctness for orchestrations of unreliable services. It has the following properties:

$$
\begin{aligned}
& P \Xi_{l} P \vee Q \\
& P \Xi_{l} P \mid Q \\
& P \vee Q \Xi_{l} P \mid Q \\
& P \downarrow_{x} \sqsubseteq_{l} P_{a}^{x} \text { for any } a \\
& 1(x)<x<P \bigsqcup_{l} P
\end{aligned}
$$

The ordering $\subseteq_{l}$ has least element false over the set of all predicates; on the set of healthy predicates 0 is the least element. Relation $\subseteq_{l}$ is reflexive and transitive but not antisymmetric: for example, the terminating orchestration

$$
\text { Term }=_{d f} \quad \theta=\{a, b\}
$$

and the potentially failing orchestration

$$
\text { Fail }=_{d f} \quad \theta=\{a, b\} \vee \theta=\{a\} \vee \theta=\{b\} \vee \theta=\{\}
$$

cannot be distinguished using $\sqsubseteq_{l}$ because Term $\sqsubseteq_{l}$ Fail $\wedge$ Fail $\sqsubseteq_{l}$ Term. Conventionally $\sqsubseteq_{l}$ is treated as an antisymmetric relation over equivalence classes of predicates: $P$ and $Q$ are in the same equivalence class, denoted as $P \equiv_{l} Q$, iff $P \sqsubseteq_{l} Q \wedge Q \sqsubseteq_{l} P$. For example,

$$
\left(\theta=\left\{\left(x, x^{2}\right),\left(x, x^{3}\right)\right\}\right) \equiv_{l} \quad\left(\theta=\{\} \vee \theta=\left\{\left(x, x^{2}\right)\right\} \vee \theta=\left\{\left(x, x^{3}\right)\right\} \vee \theta=\left\{\left(x, x^{2}\right),\left(x, x^{3}\right)\right\}\right)
$$


Theorem 5.1 The operators $\gg, \mid, \ll$ and $\triangleleft \triangleright$ are monotonic on the lower powerdomain (see Appendix for details).

The least upper bound of a partially ordered set $S$ is denoted by $\bigsqcup S$ and satisfies the following properties:

$$
\begin{aligned}
& \forall x \in S . x \sqsubseteq_{l} \bigsqcup S \\
& \left(x \in S \wedge x \sqsubseteq_{l} y\right) \Rightarrow \bigsqcup S \sqsubseteq_{l} y
\end{aligned}
$$

Lemma 5.1 The least upper bound (or join) of the set $\{P, Q\}$ is $P \vee Q$.

Proof The proof is in two parts:

$P \sqcup Q \sqsubseteq_{l} P \vee Q$

Proof:

$$
\begin{aligned}
& P \sqsubseteq_{l} P \vee Q \text { defn of } \sqsubseteq_{l} \text {, since if }(\bar{x}, \bar{\theta}) \text { satisfies } P \text { then it also satisfies } P \vee Q \\
& Q \sqsubseteq_{l} P \vee Q \text { likewise } \\
& P \sqcup Q \sqsubseteq_{l} P \vee Q \text { by (2) } \\
& P \vee Q \sqsubseteq_{l} P \sqcup Q
\end{aligned}
$$

Proof:

Consider any $(\bar{x}, \bar{\theta})$ satisfying $P \vee Q$.

If $(\bar{x}, \bar{\theta})$ satisfies $P$ then it must satisfy $P \sqcup Q$ (by (1)).

If $(\bar{x}, \bar{\theta})$ satisfies $Q$ then it must satisfy $P \sqcup Q$ (by (1)).

The results above hold for arbitrary $(\bar{x}, \bar{\theta})$ satisfying $P \vee Q$.

Therefore $P \vee Q \sqsubseteq_{l} P \sqcup Q$.

A chain is an increasing sequence of orchestrations:

$$
P_{1} \sqsubseteq_{l} P_{2} \sqsubseteq_{l} \cdots
$$

The least upper bound of a chain $P_{i}$ is denoted by $\bigsqcup_{i>0} P_{i}$ and satisfies $\forall i .\left(P_{i} \sqsubseteq_{l} \bigsqcup_{j} P_{j}\right)$ and $\left(\forall i . P_{i} \sqsubseteq_{l} x\right) \Rightarrow \bigsqcup_{i} P_{i} \sqsubseteq_{l} x$. The following fixed point result [Nel89] generalises the well known Knaster-Tarski Limit Theorem:

Theorem 5.2 (Generalised Limit Theorem) Let $f$ be a monotonic function on a partially ordered set in which every chain has a join, and let $f^{\alpha}$, for ordinal $\alpha$, be defined inductively by:

$$
f^{\alpha}={ }_{d f} \bigsqcup_{\beta<\alpha} f\left(f^{\beta}\right)
$$

$f^{0}$ is the empty join $(\perp) . f$ has a least fixed point given by $f^{\alpha}$, for some ordinal $\alpha$ (transfinite induction). In practice, for a non-limit ordinal, say $i, f^{i}=f\left(f^{i-1}\right)$-since $f_{i-1} \sqsubseteq f_{i} \Rightarrow f_{i-1} \sqcup f_{i}=f_{i}$.

Orchestrations from a partially ordered set, under the relation $\bigsqcup_{l}$, in which every chain $P_{1} \sqsubseteq_{l} P_{2} \sqsubseteq_{l} \ldots$ has a least upperbound $\bigvee_{i \geq 0} P_{i}$ (see Lemma 5.1). Thus, Theorem 5.2 can be applied to determine the least fixed point of a recursive orchestration equation.

Example 2 In the following predicates of the form $\theta=A \cup B \vee \theta=B \vee \theta=\{\}$ are simplified to $\theta=A \cup B$ since $\theta=A \cup B \equiv_{l} \quad(\theta=A \cup B \vee \theta=B \vee \theta=\{\})$. The least fixed point of $f a c$ can be calculated using the generalised limit theorem:

$$
\begin{aligned}
& f a c_{0}(x)=0 \\
& \operatorname{fac}_{i}(x)=\theta=\{x !\} \triangleleft 0 \leq x<i \triangleright \theta=\{\} \quad \forall i>0 \\
& f a c_{\omega}(x)=(\theta=\{x !\}) \triangleleft(0 \leq x) \triangleright \theta=\{\}
\end{aligned}
$$

The least fixed point of $f^{*}$ can be generated in a similar way:

$$
\begin{aligned}
f_{0}^{*} & =0 \\
f_{i}^{*}(x) & =\theta=\{f(x)\} \vee \theta=\{\} \forall i>0
\end{aligned}
$$


Thus, $f^{*}$ can diverge if an infinite series of time-outs occurs. In a similar way $S(n)$ gives rise to the following approximations:

$$
S_{i}(n)= \begin{cases}0 & \text { if } i=0 \\ (\theta=\{n, n-1, \ldots, 0\}) & \text { if } 0<n<i \\ (\theta=\{n, n-1, \ldots, n-i+1\} & \text { if } 0<i \leq n\end{cases}
$$

and

$$
\begin{aligned}
S_{\omega}(n) & =\bigvee_{i \geq 0} S_{i}(n)=(\theta=\{\} \vee \theta=\{n\} \vee \theta=\{n, n-1\} \vee \cdots \vee \theta=\{n, n-1, \ldots 0\}) \\
& \equiv_{l} \theta=\{n, n-1, \ldots 0\}
\end{aligned}
$$

The orchestration $T(n)$ has the following approximation chain:

$$
\begin{aligned}
& T_{i}(n) \equiv_{l} \begin{cases}(\theta=\{\}) & \text { if } i=0 \\
(\theta=\{y \mid n \leq y \leq n+i-1\}) & \text { if } i>0\end{cases} \\
& T_{\omega}(n)=\bigvee_{i \geq 0} T_{i+1}(n)=\exists j .(n \leq j) \wedge \theta=\{y \mid n \leq y \leq j\}
\end{aligned}
$$

Note that $\{y \mid y \geq n\}$ is also a fixed point of $T$ but that $\exists j .(n \leq j) \wedge \theta=\{y \mid n \leq y \leq j\} \sqsubseteq_{l}\{y \mid y \geq n\}$.

There are subtle distinctions between the semantics given here and a timed semantics of Orc [WKCM08]. In the later a call to service 1 responds immediately and so an evaluation of the recursive expression $T(n)$ must call $1(n)$ before $1(n+1)$. Thus, an evaluation of the expression

$$
1(z)<z<T(n)
$$

must publish the value $n$. Timing issues are not considered in this paper. Consequently, $1(z)<z<T(n)$ is identified with the predicate $\exists z . z \geq n \wedge \theta=\{z\}$ (i.e. unbounded non-determinism); here evaluation of $T(n)$ may give rise to a sequence of recursive calls to $T$ (some of which may respond) before the call to $1(n)$ responds.

Declarations can be used to define both threads (e.g. fac) and systems (e.g. T). By restricting the scope of recursive definitions it may be possible to use simpler orderings to generate approximation chains. For example, recursive thread declarations which publish at most one result may be ordered by an implication based ordering.

\section{Discussion}

In a web environment users can create applications by orchestrating a set of remote, distributed services. In this paper a basis for reasoning about orchestrations over concrete, stateless services has been proposed. The semantic framework includes a set of straightforward rules for reasoning about finite orchestrations. Partial correctness arguments are used to reason about service orchestrations in unreliable environments where failure might occur. An equivalence class $\equiv_{l}$ is employed to classify all orchestration outcomes that might arise when web-services fail. The class contains an angelic evaluation outcome, where all services are working, a daemonic evaluation outcome, where the web-environment is entirely broken, as well as a variety of less extreme scenarios. Orc declarations can be complex and may involve recursive definitions which spawn parallel threads in addition to more conventional compositional operators. It has been shown here that the operators of Orc are monotonic on the lower powerdomain and so recursive declarations can be given a fixed-point semantics.

The stateless semantics provides a tractable means of reasoning about orchestrations. The introduction of state gives rise to increased complexity. A stateful service is identified with a predicate $P\left(z, \sigma, \sigma^{\prime}, \theta\right)$ where $z$ is an input parameter, $\theta$ is a publication bag and $\sigma$ and $\sigma^{\prime}$ are initial and final states, respectively. If two services $S$ and $T$ act on a shared state $\sigma$ then the order in which $S$ and $T$ are called within the orchestration $S \mid T$ is significant. Let $S ; T$ be an orchestration which calls $S$ before $T$ and returns the combined publications of $S$ and $T$. Then the services $S(x)=_{d f} P\left(z, \sigma, \sigma^{\prime}, \theta\right)$ and $T(y)=_{d f} Q\left(z, \sigma, \sigma^{\prime}, \theta\right)$ are sequentially composed as follows:

$$
S(x) ; T(y)=_{d f} \exists \sigma_{i} . \exists b_{1} . \exists b_{2} . P\left(x, \sigma, \sigma_{i}, b_{1}\right) \wedge Q\left(y, \sigma_{i}, \sigma^{\prime}, b_{2}\right) \wedge \theta=b_{1} \cup_{b a g} b_{2}
$$

If $S$ and $T$ are atomic services then

$$
S(x) \mid T(y)=S(x) ; T(y) \vee T(y) ; S(x)
$$


However, if $S$ and $T$ are orchestrations which publish more than one value then internal state traces, for both $S$ and $T$, are needed to develop a compositional semantics for $S(x) \mid T(y)$ (and for the other Orc operators).

Services may provide "performance guarantees" through service level agreements (SLAs). Given a notion of universal time it is possible to provide response time bounds for services. Service $S(x)$ has a maximal delay $\delta(S)$, (or $\delta(S, x)$ if the delay is input dependent) if it publishes no later than time $\delta(S)$ after being called: $\delta(S) \geq 0 .{ }^{4}$ For example, $\delta(1)=0, \delta($ Google $)=\epsilon$, where $\epsilon$ is a small time bound and $\delta($ Rtimer, $t)=t$. Given a set of services $S S$ with response SLAs it is possible to give guarantees about the performance of an orchestration defined over $S S$ :

$$
\delta(P>x>Q)=\delta(P)+\delta(Q), \quad \delta(P<y<Q) \leq \delta(P)+\delta(Q)
$$

The inequality in the time bound for $P<y<Q$ arises from the (partial) overlapping of the evaluations of $P$ and $Q$ and the possibility that only a part of $Q$ is evaluated to termination in $P<y<Q$. The delay associated with parallel composition is context sensitive:

$$
\begin{aligned}
& \delta(R>(P \mid Q))=\delta((P \mid Q)>R)=\max \{\delta(P), \delta(Q)\}+\delta(R) \\
& \delta(R<y<(P \mid Q)) \leq \min \{\delta(P), \delta(Q)\}+\delta(R)
\end{aligned}
$$

The work reported in this paper makes a contribution to the creation of a basis for reasoning about the correctness of service-based computations. It is anticipated that the predicate-based reasoning framework given here can be extended in ways that allow consideration of alternative forms of orchestration in which sites or services are published.

\section{Acknowledgments}

The authors are grateful to Maurice Clint and the referees for their comments on earlier drafts of this paper. Alan Stewart was partially supported by EPSRC project EP/I03405X/1 (ECHO). Joaquim Gabarro was partially supported by FET pro-active Integrated Project 15964 (AEOLUS), the projects TIN2007-66523 (FORMALISM) and TIN2005-25859-E of "Ministerio de Ciencia e Inovación y el Fondo Europeo de Desarrollo Regional".

\section{Appendix: Properties of the Orc combinators}

Lemma $1 P \sqsubseteq_{l} P \mid Q$.

Proof $P \sqsubseteq_{l} P \mid Q \Leftrightarrow\left(\right.$ defn of $\sqsubseteq_{l}$ and $\left.\mid\right)$

$\forall \theta . P \Rightarrow \exists \theta^{\prime} . \exists r_{1} . \exists r_{2} . P_{r_{1}}^{\theta} \wedge Q_{r_{2}}^{\theta} \wedge \theta^{\prime}=r_{1} \cup_{b a g} r_{2} \wedge \theta \subseteq \subseteq_{b a g} \theta^{\prime}$ (let $\left.r_{1}=\theta\right)=$

$\forall \theta . P \Rightarrow \exists \theta^{\prime} . \exists r_{2} \cdot Q_{r_{2}}^{\theta} \wedge \theta^{\prime}=\theta \cup_{\text {bag }} r_{2} \Leftrightarrow$

$\exists r_{2} . \wedge Q_{r_{2}}^{\theta} \Leftrightarrow(Q$ is healthy $)$

true

Lemma 2 Parallel composition is monotonic on $\sqsubseteq_{l}$ :

$\left(P \sqsubseteq_{l} Q\right) \Rightarrow(P \mid R) \sqsubseteq_{l}(Q \mid R)$

Proof Assume that $P \sqsubseteq_{l} Q$

Consider $P \mid R=\exists r, r_{2} . P_{r}^{\theta} \wedge R_{r_{2}}^{\theta} \wedge \theta=r_{1} \cup r_{2}$

Consider $P_{r}^{\theta}$ : Since $P \sqsubseteq_{l} Q$ it follows that $\exists r^{\prime} . Q_{r^{\prime}}^{\theta} \wedge r \subseteq_{b a g} r^{\prime}$

And so $\exists \theta^{\prime} . \exists r^{\prime}, r_{2} \cdot Q_{r^{\prime}}^{\theta} \wedge R_{r_{2}}^{\theta} \wedge \theta^{\prime}=r^{\prime} \cup r_{2} \wedge \theta \subseteq_{b a g} \theta^{\prime}$

$\overline{4}$ In practice SLAs provide response time bounds which are applicable for only a certain percentage of service calls. 
Lemma $3 \forall b . b \subseteq b^{\prime} \Rightarrow R_{b}^{x} \sqsubseteq_{l} R_{b^{\prime}}^{x}$

Proof Proof by induction over $b^{\prime}$ :

Base case: $b^{\prime}=\{\}$ : the result follows since $b=b^{\prime}$.

Inductive case: assume that $\forall b . b \subseteq b^{\prime} \Rightarrow R_{b}^{x} \sqsubseteq_{l} R_{b^{\prime}}^{x}$

Consider: $\forall b . b \subseteq b^{\prime} \cup\{v\} \Rightarrow R_{b}^{x} \sqsubseteq_{l} R_{b^{\prime} \cup\{v\}}^{x}$

case $1 . b \subseteq b^{\prime}$ :

$$
R_{b}^{x} \sqsubseteq_{l} R_{b^{\prime}}^{x} \text { (by assumption) } \sqsubseteq_{l} R_{b^{\prime}}^{x} \mid R_{v}^{x}=R_{b^{\prime} \bigsqcup_{b a g}\{v\}}^{x} \text { (since } P \sqsubseteq_{l} P \mid Q \text { ) }
$$

case 2. $b-\{v\} \subseteq b^{\prime}$

$$
R_{b-\{v\}}^{x} \sqsubseteq_{l} R_{b^{\prime}}^{x} \text { (by assumption). }
$$

Thus $R_{b-\{v\}}^{x}\left|R_{v}^{x} \sqsubseteq_{l} R_{b^{\prime}}^{x}\right| R_{v}^{x}$ (monotonicity of $\mid$ ) and so $R_{b}^{x} \sqsubseteq_{l} R_{b^{\prime} \cup\{v\}}^{x}$

Lemma $4 P \sqsubseteq Q \Rightarrow P_{b}^{x} \sqsubseteq l Q_{b}^{x}$.

Proof Proof by induction over $b$ :

Base case: $b=\{\}:$ the result follows since $P_{b}^{x}=0=Q_{b}^{x}$.

Inductive case: Assume that $P \sqsubseteq Q \Rightarrow P_{b}^{x} \sqsubseteq_{l} Q_{b}^{x}$. Consider the bag $b \cup\{v\}$ then

$$
P_{b \cup\{v\}}^{x}=P_{b}^{x}\left|P_{v}^{x} \sqsubseteq_{l} Q_{b}^{x}\right| P_{v}^{x} \sqsubseteq_{l} Q_{b}^{x} \mid Q_{v}^{x}=Q_{b \cup\{v\}}^{x}
$$

Lemma 5 Sequential composition is monotonic on $\sqsubseteq_{l}$. The proof is in two parts:

$\left(P \sqsubseteq_{l} Q\right) \Rightarrow(P>x>R) \sqsubseteq_{l}(Q>x>R)$ and

$\left(P \sqsubseteq_{l} Q\right) \Rightarrow(R>x>P) \sqsubseteq_{l}(R>x>Q)$

Proof Part 1. Assume that $P_{b}^{\theta}$ holds.

Since $P \sqsubseteq_{l} Q$ then $\exists b^{\prime} . Q_{b^{\prime}}^{\theta} \wedge b \subseteq_{b a g} b^{\prime}$

But, $P>x>R=R_{b}^{x}$ and $Q>x>R=R_{b^{\prime}}^{x}$

The result follows by applying Lemma 3. Part 2. $\left(P \sqsubseteq_{l} Q\right) \Rightarrow(R>x>P) \sqsubseteq_{l}(R>x>Q)$

The result follows directly from Lemma 4.

Lemma 6 Pruning composition is monotonic on $\sqsubseteq_{l}$ :

The proof is in two parts:

$\left(P \sqsubseteq_{l} Q\right) \Rightarrow\left((R<z<P) \sqsubseteq_{l}(R<z<Q)\right)$

$\left(P \sqsubseteq_{l} Q\right) \Rightarrow\left((P<z<R) \sqsubseteq_{l}(Q<z<R)\right)$

Proof Part 1. The proof is bay case analysis:

case 1: $P=0$ :

$$
\left.(R<z<P)=R \downarrow_{x} \sqsubseteq_{l} \quad(R<z<Q) \quad \text { (for any } Q\right)
$$

case $2: \exists \theta \cdot P \wedge \theta \neq\{\}$ :

Then $\exists \theta^{\prime} . Q_{\theta^{\prime}}^{\theta} \wedge \theta \sqsubseteq_{l} \theta^{\prime}$ (since $P \sqsubseteq_{l} Q$ ).

If $v \in \theta$ then $v \in \bar{\theta}^{\prime}$ (since $\theta \subseteq$ bag $\theta^{\prime}$ ).

Hence $\left(\exists v . v \in \theta \wedge R_{v}^{z}\right) \sqsubseteq_{l}\left(\exists v \cdot v \in \theta^{\prime} \wedge R_{v}^{z}\right)$.

Part 2.

$\left(P \sqsubseteq_{l} Q\right) \Rightarrow\left((P<z<R) \sqsubseteq_{l}(Q<z<R)\right)$

case 1: $R=0$ :

Then $(P<z<R)$ and $(Q<z<R)$ behave as $P \downarrow_{x}$ and $Q \downarrow_{x}$, respectively.

But $P \downarrow_{x} \sqsubseteq_{l} Q \downarrow_{x}$ since $\left(P \sqsubseteq_{l} Q\right)$.

case $2: \exists b . \exists v \cdot v \in b \wedge R_{b}^{\theta}$ :

In this case $(P<z<R)$ and $(Q<z<R)$ can behave as $P_{v}^{z}$ and $Q_{v}^{z}$, respectively, for any $v \in b$.

But $P_{v}^{z} \sqsubseteq_{l} Q_{v}^{z}\left(\right.$ since $\left.P \sqsubseteq_{l} Q\right)$. 
Lemma 7 The operation $\triangleleft \triangleright$ is monotonic on $\sqsubseteq_{l}$.

Proof The proof is in two parts:

$\left(P \sqsubseteq_{l} Q\right) \Rightarrow\left(R \triangleleft b \triangleright P \sqsubseteq_{l} R \triangleleft b \triangleright Q\right)$

If $b$ holds the result follows since $R \sqsubseteq_{l} R$.

If $\neg b$ holds the result follows since $P \sqsubseteq_{l} Q$.

A proof that $\left(P \sqsubseteq_{l} Q\right) \Rightarrow\left(P \triangleleft b \triangleright R \sqsubseteq_{l} Q \triangleleft b \triangleright R\right)$ follows in a similar way.

\section{References}

[BvW] Back R-J, von Wright J (1998) Refinement calculus: a systematic introduction. Springer, New York

[Bk09] Back R-J (2009) Invariant based programming: basic approach and teaching experiences. Form Asp Comp 21(3):227-244

[BBGGS07] Baresi L, Bianculli D, Ghezzi C, Guinea S, Spoletini P (2007) Validation of web service compositions. IET Softw 1(6):219-232

[Dij76] Dijkstra EW (1976) A discipline of programming. Prentice-Hall, Englewood Cliffs

[DLSZ06] Dong JS, Liu Y, Sun J, Zhang X (2006) Verification of computation orchestration via timed automata. In: Liu Z, He J (eds) Formal methods and software engineering. LNCS, vol 4260, pp 226-245

[GS90] Gunter CA, Scott DS (1990) Semantic domains, In: van Leeuvan J (ed) Handbook of theoretical computer science: formal models and semantics. Elsevier. pp 633-674

[Heh84a] Hehner ECR (1984) Predicative programming part I. Commun ACM 27(2):134-143

[Heh84b] Hehner ECR (1984) Predicative programming part II. Commun ACM 27(2):144-151

[Hoa69] Hoare CAR (1969) An axiomatic basis for computer programming. Commun ACM 12(10):576-580

[Hoa85] Hoare CAR (1985) Programs are predicates. In: Hoare CAR, Shepherdson JC (eds) Mathematical logic and programming languages. Prentice-Hall, pp 141-155

[Hoa85] Hoare CAR (1985) Communicating sequential processes. Prentice Hall

[HH98] Hoare CAR, He J (1998) Unifying theories of programming. Prentice Hall

[HMM04] Hoare CAR, Menzel G, Misra J (2004) A tree semantics of an orchestration language. In: Broy M (ed) Proc of the NATO Advanced Study Institute, Engineering Theories of Software Intensive Systems. NATO ASI Series Marktoberdorf, Germany

[KCM06] Kitchin D, Cook WR, Misra J (2006) A language for task orchestration and its semantic properties. In: Baier C, Hermanns $\mathrm{H}$ (eds) Proc of the international conference on concurrency theory (CONCUR). LNCS, vol 4137, pp 477-491

[Kit09] Kitchin D, Quark A, Cook W, Misra J (2009) The Orc programming language. Proceedings of FMOODS/FORTE. LNCS, vol 5522, pp 1-25. Springer

[KPM08] Kitchin D, Powell E, Misra J (2008) Simulation using orchestration. In: Meseguer J, Rou G (eds) Proceedings of the 12th international conference on algebraic methodology and software technology 2008. LNCS, vol 5140, pp 2-15

[LZH10] Li Q, Zhu H, He J (2010) A denotational semantical model for Orc language. In: Cavalcanti A, Deharbe D, Gaudel M-C, Woodcock J (eds) Theoretical aspects of computing. ICTAC 2010. LNCS, vol 6255, pp 106-120

[NS10] Njima NM, Sanders JW (2010) Specification-oriented orchestration. In: Proceedings of the 2010 international conference on communications and mobile computing, vol 01. IEEE, pp 550-554

[Mis04] Misra J (2004) Computation orchestration: a basis for a wide-area computing. In: M Broy (ed) Proceedings of the NATO Advanced Study Institute, Engineering Theories of Software Intensive Systems. NATO ASI Series Marktoberdorf, Germany

[MC07] Misra J, Cook WR (2007) Computation orchestration: a basis for wide-area computing. J Softw Syst Model 6(1):83-110

[Nel89] Nelson G (1989) A generalization of Dijkstra's calculus: ACM Trans Program Lang Syst 11:517-561

[SGC06] Stewart A, Gabarró J, Clint M, Harmer T, Kilpatrick P, Perrott R (2006) Managing grid computations: an Orc-based approach. In: Guo M et al (eds) Parallel and distributed processing and applications (ISPA 2006). LNCS, vol 4330, pp 278-291

[VW08] Vardoulakis D, Wand M (2008) A compositional trace semantics for Orc. In: Coordination models and languages. LNCS, vol 5052. Springer, pp 331-346

[WKCM08] Wehrman I, Kitchin D, Cook WR, Misra J (2008) A timed semantics of Orc. Theor Comput Sci 402:234-248

Received 20 November 2009

Revised 16 August 2011

Accepted 10 October 2011 by Jim Woodcock

Published online 16 November 2011 